\title{
Possible tumor suppressive role of the miR-144/451 cluster in esophageal carcinoma as determined by principal component regression analysis
}

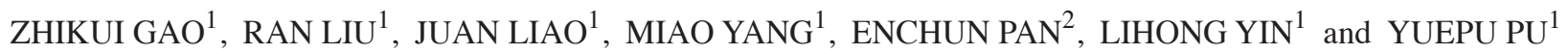 \\ ${ }^{1}$ Key Laboratory of Environmental Medicine Engineering, Ministry of Education, School of Public Health, Southeast University, \\ Nanjing, Jiangsu 210009; ${ }^{2}$ Huaian Center for Disease Control and Prevention, Huaian, Jiangsu 223001, P.R. China
}

Received September 4, 2015; Accepted June 24, 2016

DOI: $10.3892 / \mathrm{mmr} .2016 .5691$

\begin{abstract}
MicroRNA (miRNA) clusters are expressed universally across different types of organisms, and an accumulating number of studies have demonstrated that miRNA clusters function more efficiently compared with single miRNAs during the development of certain cancer types. miRNA clusters may have increased stability and reliability over individual miRNAs as diagnostic or therapeutic biomarkers. In the present study, the expression levels of mature miRNAs within the miR-144/451 cluster were examined using stem-loop reverse transcription-quantitative polymerase chain reaction in 102 patients pathologically diagnosed with esophageal carcinoma. Bioinformatics tools were used to identify a possible miRNA-mediated network of the miR-144/451 cluster. The expression levels of hsa-miR-451a, hsa-miR-144-3p and hsa-miR-144-5p in tumor tissues were significantly lower compared with those in adjacent non-tumor tissues $(\mathrm{P}<0.05)$. Pearson correlation analysis demonstrated that the expression levels of individual miR-144/451 cluster members were correlated with each other, except for the pair of hsa-miR-144-3p and hsa-miR-4732-3p. In particular, hsa-miR-144-5p expression was highly associated with hsa-miR-4732-5p and hsa-miR-451a expression levels, with correlation coefficients of 0.729 and 0.608 , respectively. Furthermore, the low expression levels of hsa-miR-144-3p [odds ratio (OR), 0.85; $\mathrm{P}<0.05$ ] and hsa-miR-144-5p (OR, $0.84 ; \mathrm{P}<0.05)$ were determined to be risk factors for esophageal carcinoma development. Kyoto Encyclopedia of Genes and Genomes pathway analysis demonstrated that miRNAs forming the miR-144/451 cluster may cooperate to regulate
\end{abstract}

Correspondence to: Professor Ran Liu, Key Laboratory of Environmental Medicine Engineering, Ministry of Education, School of Public Health, Southeast University, 87 Dingjiaqiao Street, Nanjing, Jiangsu 210009, P.R. China

E-mail:ranliu@seu.edu.cn

Key words: esophageal carcinoma, microRNA-144/451 cluster, biomarker, diagnosis the cell cycle. Therefore, the miR-144/451 cluster may serve an important role in the progression of esophageal carcinoma and may be considered as a biomarker for the detection of esophageal carcinoma at an early stage.

\section{Introduction}

Esophageal carcinoma is a common type of cancer of the digestive system, with an estimated 456,000 new cases and 400,000 cases of mortality worldwide during 2012, and with $50 \%$ of all cases occurring in China alone $(1,2)$. In patients receiving early comprehensive treatment, the 5-year relative survival rate of esophageal carcinoma approaches $90 \%$. However, the overall 5 -year survival rate of patients with esophageal carcinoma remains poor as it is most commonly diagnosed at an advanced stage (with lymph node metastases) and only few symptoms appear at an early stage $(3,4)$. Therefore, there is an urgent requirement for novel and reliable biomarkers that facilitate the detection of preneoplastic esophageal carcinoma lesions.

Typically, microRNA (miRNA) genes are transcribed under the regulation of a promoter and an operon. Certain miRNAs are positioned proximal to each other on the chromosome and form a cluster (5-7). These clusters are considered to be transcribed simultaneously, but may mediate synergistic or antagonistic regulatory functions $(8,9)$. An increasing number of studies have demonstrated that miRNA clusters serve important roles in certain biological processes and during oncogenesis (10-13). A few well-studied miRNA clusters include the miR-17/92, miR-221/222 and miR-1/133a clusters (14-16). Additional studies have demonstrated that miRNA clusters function more efficiently compared with individual miRNAs alone. For instance, Li et al (17) demonstrated that miR-424 and miR-503, forming the miR-424-503 cluster, cooperate to inhibit the expression of Smad7 and Smurf2, thus increasing the transforming growth factor- $\beta$ signaling and the metastatic potential of breast cancer cells. In addition, Wystub et al (18) observed that miR-1 and miR-133a, forming the miR-1/133a cluster, cooperate to modulate the cardiomyogenic lineage by suppressing the expression levels of MYOCD and KCNMB1 genes, respectively. Previous miRNA profiling data demonstrated that two members of the miR-144/451 cluster, namely miR-144-3p 
and miR-451a, were significantly downregulated in tumor tissues $(\mathrm{P}=0.039$ and 0.005 , respectively) compared with adjacent non-tumor tissues (19). Furthermore, studies have provided evidence to suggest that miR-144-3p and miR-451a may serve tumor suppressive or oncogenic roles in different tissues $(16,20,21)$.

The majority of studies investigating the miR-144/451 cluster have focused on elucidating the function of the individual miRNAs, but not the cluster as a whole. In addition, there is currently no detailed information about the miR-144/451 cluster in esophageal carcinoma (22-24). It is possible that individual miRNAs within the miR-144/451 cluster may mediate opposing or similar functions as part of complex regulatory networks. Clustered miRNAs appear to be more stable and reliable than individual miRNAs as diagnostic biomarkers $(18,19,25)$. In addition, detailed information on the expression levels and functional roles of the miR-144/451 cluster may facilitate an improved understanding of the mechanisms involved in tumor development and maintenance, which may also be beneficial for therapy. Promoting or suppressing the expression of miRNAs in the miR-144/451 cluster may be a promising therapeutic strategy, with high efficiency and low treatment resistance, for patients with esophageal carcinoma.

In the present study, the expression levels of miR-144/451 cluster members in esophageal carcinoma tissues were examined, and the correlation between the expression levels of individual miRNAs were determined. To the best of our knowledge, this is the first study that has reported the expression levels of hsa-miR-144-3p, hsa-miR-144-5p, hsa-miR-451a, hsa-miR-4732-3p and hsa-miR-4732-5p, which comprise the miR-144/451 cluster, in esophageal carcinoma. Pearson correlation analyses were performed to evaluate the association between the expression levels of all five miRNAs. The association between abnormal expression of the miR-144-451 cluster and the risk of esophageal carcinoma was further analyzed using principal component regression analysis. The possible targets and functions of the miR-144-451 cluster were analyzed by bioinformatics. The results of the present study may advance our understanding of the expression pattern and functional role of the miR-144/451 cluster in esophageal carcinoma.

\section{Materials and methods}

Study subjects. Samples from 102 patients with esophageal carcinoma, diagnosed by pathological analyses (26) and recruited from the First People's Hospital of Huaian (Huaian, China), were used in the present study. Among these patients, $73(71.6 \%)$ cases were male and $29(28.4 \%)$ were female, with an age range of 44-78 years and a mean age of $63.17 \pm 7.25$ years. Esophageal carcinoma tissues and their corresponding normal non-tumor tissues were collected surgically between 2011 and 2012 , and stored in tubes at $-80^{\circ} \mathrm{C}$. Written informed consent was obtained from all subjects prior to recruitment to the study. Ethical approval was provided by the Institutional Review Board of the Southeast University-Affiliated Zhongda Hospital (Nanjing, China).

RNA isolation and analysis. Total RNA was extracted from the tissue samples using the TRIzol reagent (Invitrogen; Thermo Fisher Scientific, Inc., Waltham, MA, USA) according to the manufacturer's instructions. The quality and concentration of the extracted RNA was assessed with the 260/280 absorbance ratio using the NanoDrop ND-1000 spectrophotometer (Thermo Fisher Scientific, Inc.).

Reverse transcription-quantitative polymerase chain reaction $(R T-q P C R)$. Bulge-Loop ${ }^{\mathrm{TM}}$ miRNA RT-qPCR Primer kits (catalog nos. MQP-0101 and MQP-0201) were obtained from Guangzhou RiboBio Co., Ltd. (Guangzhou, China). A total of $0.5 \mu \mathrm{g}$ RNA template, $1 \mu \mathrm{l}$ miRNA-specific stem-loop RT-primers and RNase-free water (Tiangen Biotech, Co., Ltd., Beijing, China) were combined. Next, $5.5 \mu \mathrm{l}$ mixtures were incubated at $70^{\circ} \mathrm{C}$ for $10 \mathrm{~min}$, and then immediately placed on ice for $2 \mathrm{~min}$. Reverse transcription of RNA into cDNA was subsequently performed by addition of $2.5 \mu 15 \mathrm{X}$ RT buffer (Promega Corp., Madison, WI, USA), $1 \mu \mathrm{l}$ dNTPs ( $2.5 \mathrm{mM}$; Tiangen Biotech, Co., Ltd.), $0.25 \mu \mathrm{l}$ M-MLV reverse transcriptase (200 U/ $\mu \mathrm{l}$; Promega Corp.), $0.25 \mu 1$ ribonuclease inhibitor (40 U/ $\mu \mathrm{l}$; Fermentas; Thermo Fisher Scientific, Inc.) and $3 \mu \mathrm{l}$ RNase-free water. The sample was subsequently incubated for $1 \mathrm{~h}$ at $42^{\circ} \mathrm{C}$, followed by $70^{\circ} \mathrm{C}$ for $10 \mathrm{~min}$ and then held at $4^{\circ} \mathrm{C}$. qPCR was performed using the StepOnePlus system (Applied Biosystems; Thermo Fisher Scientific, Inc.), and U6 expression (Guangzhou RiboBio Co., Ltd.) was selected to normalize the RNA input. cDNA ( $1 \mu \mathrm{l})$ was added to a mixture containing $4.5 \mu 1$ SYBR Green PCR Master Mix (Toyobo Co., Ltd., Osaka, Japan), $0.8 \mu \mathrm{l}$ forward primer, $0.8 \mu \mathrm{l}$ reverse primer and $3.7 \mu \mathrm{l}$ RNase-free water to a total volume of $10 \mu 1$. The thermal cycling conditions consisted of an initial denaturation step at $95^{\circ} \mathrm{C}$ for $5 \mathrm{~min}$, followed by 40 cycles of $95^{\circ} \mathrm{C}$ for $15 \mathrm{sec}, 60^{\circ} \mathrm{C}$ for $30 \mathrm{sec}$ and $72^{\circ} \mathrm{C}$ for $30 \mathrm{sec}$. Fluorescence data were collected at $60^{\circ} \mathrm{C}$ during each cycle, while melting curve analysis was conducted from 60 to $95^{\circ} \mathrm{C}$. The $\Delta \Delta \mathrm{C}_{\mathrm{q}}$ formula was used to quantify the relative target miRNA expression levels (the fold change in target gene expression was equal to $2^{-\Delta \Delta \mathrm{Cq}}$ ) (27).

Bioinformatics analysis. TargetScan (www.targetscan.org/), miRDB (www.mirdb.org/), PicTar (pictar.mdc-berlin.de/) and miRanda (www.microrna.org/) software were used to predict the potential targets of hsa-miR-144-3p, hsa-miR-144-5p and hsa-miR-451a. The Database for Annotation, Visualization and Integrated Discovery (DAVID) Bioinformatics Resources 6.7 (david.abcc.ncifcrf.gov/) was used for functional enrichment analysis. In addition, the Kyoto Encyclopedia of Genes and Genomes (KEGG) database (www.kegg.jp/kegg/pathway. html) was used for pathway analysis.

Statistical analysis. Statistical analysis of the data was performed using SAS version 9.2 software (SAS Institute, Cary, NC, USA). Any data records with missing values were excluded from the analysis. The following statistical tests were used to analyze the data: Conditional logistic regression, Pearson correlation, principal component analysis, multiple logistic regression and paired $t$-test. $\mathrm{P}<0.05$ was considered to indicate a statistically significant difference.

\section{Results}

Expression levels of miR-144/451 cluster members in esophageal carcinoma. The paired sample $t$-test was used to compare 
Table I. Expression levels of miRNA members of the miR-144/451 cluster in esophageal carcinoma and adjacent non-tumor tissue samples.

\begin{tabular}{|c|c|c|c|c|c|c|c|}
\hline miRNA & Group & Sample no. & $\Delta \mathrm{C}_{\mathrm{q}}$ & $\Delta \Delta \mathrm{C}_{\mathrm{q}}$ & $2^{-\Delta \Delta C q}$ & t-value & $\mathrm{P}$-value \\
\hline \multirow[t]{2}{*}{ hsa-miR-144-3p } & $\mathrm{T}$ & 101 & $19.090 \pm 2.459$ & $1.412 \pm 1.760$ & 0.376 & 8.062 & $<0.001$ \\
\hline & $\mathrm{N}$ & 101 & $17.678 \pm 2.788$ & & & & \\
\hline \multirow[t]{2}{*}{ hsa-miR-144-5p } & $\mathrm{T}$ & 99 & $17.522 \pm 2.239$ & $1.111 \pm 1.764$ & 0.463 & 6.267 & $<0.001$ \\
\hline & $\mathrm{N}$ & 99 & $16.411 \pm 2.301$ & & & & \\
\hline \multirow[t]{2}{*}{ hsa-miR-451a } & $\mathrm{T}$ & 99 & $9.300 \pm 2.666$ & $1.176 \pm 1.843$ & 0.443 & 6.348 & $<0.001$ \\
\hline & $\mathrm{N}$ & 99 & $8.124 \pm 3.057$ & & & & \\
\hline \multirow[t]{2}{*}{ hsa-miR-4732-5p } & $\mathrm{T}$ & 100 & $17.716 \pm 3.191$ & $-0.161 \pm 1.845$ & 1.118 & -0.871 & 0.386 \\
\hline & $\mathrm{N}$ & 100 & $17.876 \pm 2.858$ & & & & \\
\hline \multirow[t]{2}{*}{ hsa-miR-4732-3p } & $\mathrm{T}$ & 93 & $19.780 \pm 2.281$ & $-0.041 \pm 2.311$ & 1.029 & -0.171 & 0.865 \\
\hline & $\mathrm{N}$ & 93 & $19.821 \pm 2.376$ & & & & \\
\hline
\end{tabular}

Values are presented as the mean \pm standard deviation. $\mathrm{P}<0.001$ vs. adjacent non-tumor tissues. miR, microRNA; $\mathrm{T}$, tumor tissues; $\mathrm{N}$, adjacent non-tumor tissues; $\Delta \mathrm{C}_{\mathrm{q}}=\mathrm{C}_{\mathrm{q}(\text { target gene })} \mathrm{C}_{\mathrm{q}(\mathrm{U} 6)} ; \Delta \Delta \mathrm{C}_{\mathrm{q}}=\Delta \mathrm{C}_{\mathrm{q}(\mathrm{T} \text { group })}-\Delta \mathrm{C}_{\mathrm{q}(\mathrm{N} \text { group })}$.

the mean miRNA expression levels between esophageal tumor and adjacent non-tumor tissues (Table I). The results indicated that hsa-miR-144-3p, hsa-miR-144-5p and hsa-miR-451a were significantly downregulated in tumor tissues compared with the adjacent non-tumor tissues ( $\mathrm{P}<0.001$ for all three miRNAs) and the fold-change $\left(2^{-\Delta \Delta \mathrm{Cq}}\right)$ was found to be $0.376,0.463$ and 0.443 , respectively. By contrast, no statistically significant differences in hsa-miR-4732-5p and hsa-miR-4732-3p expression levels were observed between esophageal carcinoma and adjacent non-tumor tissues.

Correlation analysis of the expression levels of $\mathrm{miR}-144 / 451$ cluster members. Pearson correlation analysis was performed to estimate the degree of association between the members of the miR-144/451 cluster. A significant correlation was observed among the relative expression levels $\left(\Delta \mathrm{C}_{\mathrm{q}}\right)$ of the majority of miRNA members. Notably, strong correlations were observed among the expression levels of hsa-miR-144-5p, hsa-miR-4732-5p and hsa-miR-451a, with the correlation coefficients of two canonical pairs (miR-144-5p and miR-451a; miR-4732-5p and miR-451a) being $0.729(\mathrm{P}<0.001)$ and $0.608(\mathrm{P}<0.001)$, respectively (Fig. 1). However, no significant correlation was detected between the expression levels of hsa-miR-144-3p and hsa-miR-4732-3p.

Association between the expression levels of miR-144/451 cluster members and the risk of esophageal carcinoma. Conditional logistic regression models that coped with 1:1 case-control matching were used to analyze the association between the abnormal expression levels of miR-144/451 cluster members and the risk for esophageal carcinoma (Table II). The results demonstrated that reduced hsa-miR-144-5p, hsa-miR-144-3p and hsa-miR-451a expression levels were significantly associated with an increased risk for esophageal cancer [odds ratio (OR), 0.494, 0.370 and 0.474 , respectively]; however, no association was observed for hsa-miR-4732-5p and hsa-miR-4732-3p. Therefore,
hsa-miR-144-5p, hsa-miR-144-3p and hsa-miR-451a may serve important roles in the progression of esophageal carcinoma, which requires further validation in future studies.

Principal component analysis. In order to eliminate multicollinearity of individual miRNAs in the miR-144/451 cluster, principal component analysis was performed to select the principal components of clustered miRNAs (Table III). Two principal components were extracted, and their cumulative contribution of variance accounted for $70 \%$, which is sufficient to reflect the original factor's information of the cluster. The results demonstrated that hsa-miR-4732-5p, hsa-miR-451a and hsa-miR-144-5p primarily contributed to the F1 value, with scoring coefficients of $0.788,0.801$ and 0.891 , respectively (Table IV).

Multiple logistic regression analysis. Considering the strong correlation among the expression levels of hsa-miR-4732-5p, hsa-miR-451a and hsa-miR-144-5p, these miRNAs were grouped together and represented as F-miRNAs. Multiple logistic regression analysis was conducted on F-miRNAs, as well as hsa-miR-144-3p, in order to verify the previous findings (Table V). The OR values for hsa-miR-144-3p and F-miRNAs were 0.85 and 0.84 , respectively, while the downregulation of hsa-miR-144-3p and F-miRNA expression levels was found to be associated with an increased risk of esophageal carcinoma development. These results suggest that the miR-144/451 cluster may serve as a biomarker for the early detection of esophageal carcinoma.

Target prediction for selected miRNAs. The TargetScan, miRDB, PicTar and miRanda algorithms were used to predict the potential targets of hsa-miR-144-3p, hsa-miR-144-5p and hsa-miR-451a, which were abnormally expressed in esophageal carcinoma tissues. Targets identified by more than two prediction tools were selected for further analysis. For hsa-miR-144-3p, hsa-miR-144-5p and hsa-miR-451a, a total of 88,149 and 56 potential targets were selected, respectively. 
Table II. Conditional logistic regression analysis to determine the association between the expression levels of miR-144/451 cluster members and esophageal carcinoma risk.

\begin{tabular}{|c|c|c|c|c|c|c|c|}
\hline miRNA & Group & $\beta$ & SE & Wald & P-value & OR & $95 \% \mathrm{CI}$ \\
\hline \multirow[t]{2}{*}{ hsa-miR-4732-5p } & $\mathrm{T}$ & 0.096 & 0.110 & 0.753 & 0.386 & 1.100 & $0.887-1.365$ \\
\hline & $\mathrm{N}$ & & & & & 1 & \\
\hline \multirow[t]{2}{*}{ hsa-miR-144-5p } & $\mathrm{T}$ & -0.706 & 0.152 & 21.658 & $<0.001$ & 0.494 & $0.367-0.665$ \\
\hline & $\mathrm{N}$ & & & & & 1 & \\
\hline \multirow[t]{2}{*}{ hsa-miR-144-3p } & $\mathrm{T}$ & -0.995 & 0.191 & 27.055 & $<0.001$ & 0.370 & $0.254-0.538$ \\
\hline & $\mathrm{N}$ & & & & & 1 & \\
\hline \multirow[t]{2}{*}{ hsa-miR-451a } & $\mathrm{T}$ & -0.748 & 0.159 & 22.137 & $<0.001$ & 0.474 & $0.347-0.647$ \\
\hline & $\mathrm{N}$ & & & & & 1 & \\
\hline \multirow[t]{2}{*}{ hsa-miR-4732-3p } & $\mathrm{T}$ & 0.016 & 0.090 & 0.030 & 0.864 & 1.015 & $0.851-1.212$ \\
\hline & $\mathrm{N}$ & & & & & 1 & \\
\hline
\end{tabular}

miR, microRNA; T, tumor tissues; N, adjacent non-tumor tissues; SE, standard error; OR, odds ratio; CI, confidence interval.

Table III. Principal component analysis of miR-144/451 cluster members.

\begin{tabular}{|c|c|c|c|c|c|c|}
\hline \multirow[b]{2}{*}{ Factor } & \multicolumn{3}{|c|}{ Initial eigenvalue } & \multicolumn{3}{|c|}{ Extracted eigenvalue } \\
\hline & Eigenvalue & Difference & Cumulative & Eigenvalue & Difference & Cumulative \\
\hline $\mathrm{F} 1$ & 2.443 & 0.489 & 0.489 & 2.443 & 0.489 & 0.489 \\
\hline $\mathrm{F} 2$ & 1.103 & 0.221 & 0.710 & 1.103 & 0.221 & 0.710 \\
\hline F3 & 0.739 & 0.148 & 0.858 & & & \\
\hline F4 & 0.515 & 0.103 & 0.961 & & & \\
\hline F5 & 0.201 & 0.040 & 100.0 & & & \\
\hline
\end{tabular}

miR, microRNA.

The analysis did not identify any common potential targets of all three miRNAs or more than two common potential targets between each pair of miRNAs (Fig. 2).

Enrichment analysis for selected miRNAs. Enrichment analysis of gene ontology terms for potential targets of hsa-miR-144-3p, hsa-miR-144-5p and hsa-miR-451a was performed using the DAVID Bioinformatics Resources 6.7 database, and the Benjamini-Hochberg test indicated a false discovery rate value of 0.01 . The enriched functions were ranked according to the number of predicted targets of the selected miRNAs. The most enriched function associated with hsa-miR-144-3p and hsa-miR-144-5p was the regulation of transcription, whereas cell proliferation was the most enriched function for hsa-miR-451a (Table VI).

Pathway of potential targets of selected miRNAs. Pathway analysis was performed using the KEGG pathway database, and the Benjamini-Hochberg test indicated a false discovery rate value of 0.2. Notably, a number of cell cycle regulators, including SFRP1, SFRP4, YWHAZ, YWHAB, CDKN2B, CDKN2D and STAG1, were found to be targeted by miR-451a, miR-144-5p and miR-144-3p (Fig. 3).
Table IV. Component matrix for the principal component analysis.

\begin{tabular}{lcr}
\hline Load factor & F1 & \multicolumn{1}{c}{ F2 } \\
\hline miR-4732-5p & 0.788 & 0.122 \\
miR-451a & 0.801 & -0.012 \\
miR-144-5p & 0.891 & -0.012 \\
miR-144-3p & 0.537 & -0.624 \\
miR-4732-3p & 0.311 & 0.835 \\
\hline
\end{tabular}

miR, microRNA.

\section{Discussion}

The vast majority of identified miRNA clusters are intragenic and commonly derived from polycistronic mRNA sequences, which are considered to be transcribed as independent units (28-30). The miR-144/451 cluster gene is located on chromosome 17q11.2 and encodes hsa-miR-144-3p, hsa-miR-144-5p, hsa-miR-451a, hsa-miR-4732-3p and 

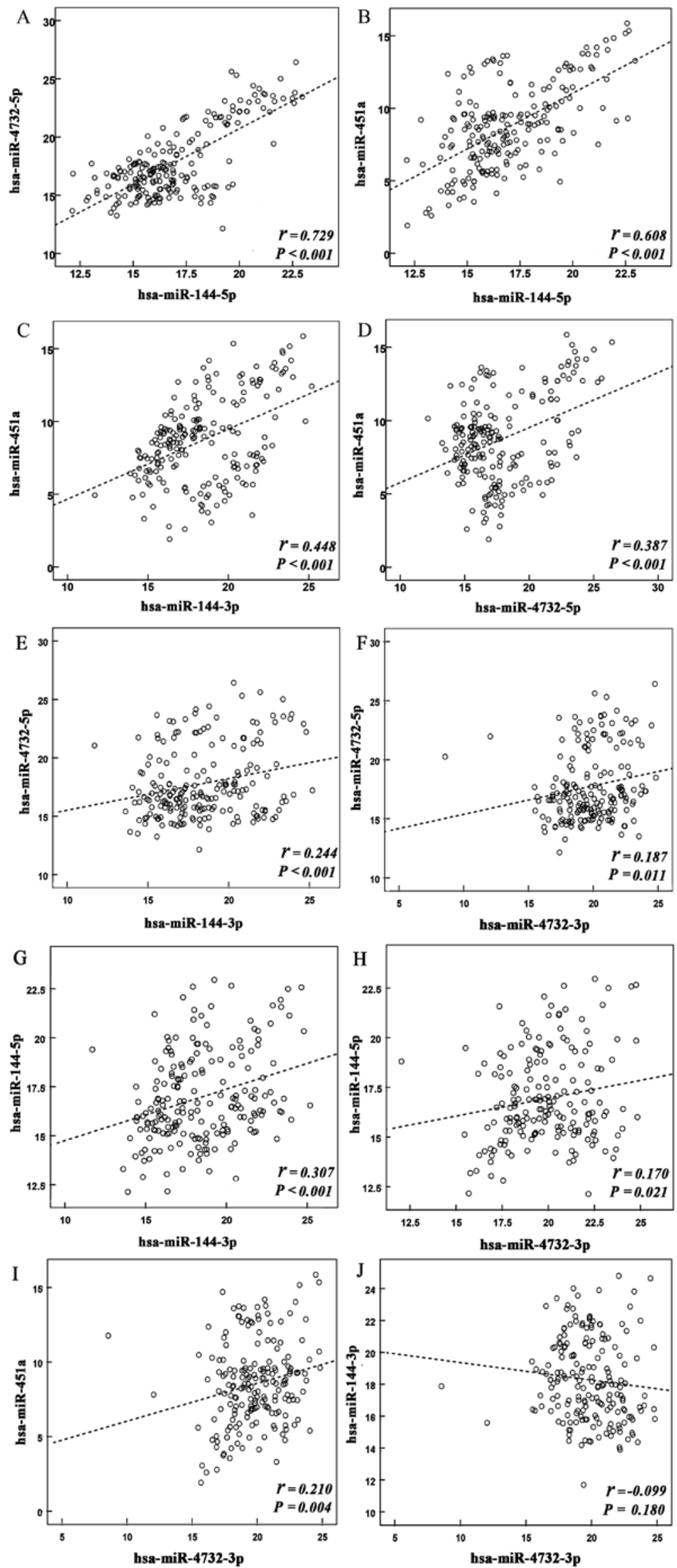

Figure 1. Correlation between the expression levels of individual miRNAs in the miR-144/451 cluster. Scatter diagrams demonstrate the association between the expression levels of (A) hsa-miR-4732-5p and hsa-miR-144-5p, (B) hsa-miR-451a and hsa-miR-144-5p, (C) hsa-miR-451a and hsa-miR-144-3p, (D) hsa-miR-451a and hsa-miR-4732-5p, (E) hsa-miR-144-3p and hsa-miR-4732-5p, (F) hsa-miR-4732-3p and hsa-miR-4732-5p, (G) hsa-miR-144-3p and hsa-miR-144-5p, (H) hsa-miR-4732-3p and hsa-miR-144-5p, (I) hsa-miR-4732-3p and hsa-miR-451a, and (J) hsa-miR-4732-3p and hsa-miR-144-3p. The axes represent the $\triangle \mathrm{Cq}$ of the relative expression levels of miRNAs. R2 values for each scatter diagram are shown. miR, microRNA. 
Table V. Multiple logistic regression analysis for the miR-144/451 cluster.

\begin{tabular}{lcccrrr}
\hline miRNA & $\beta$ & SE & Wald & P-value & OR & $95 \%$ CI \\
\hline hsa-miR-144-3p $^{*}-0.161$ & 0.059 & 7.566 & 0.006 & 0.850 & $0.757-0.954$ \\
F-miRNAs $^{\mathrm{a}}$ & -0.175 & 0.070 & 6.300 & 0.012 & 0.840 & $0.733-0.962$ \\
Constant & 5.946 & 1.431 & 17.272 & $<0.001$ & 382.204 & \\
\hline
\end{tabular}

${ }^{\mathrm{a}} \mathrm{F}-\mathrm{miRNAs}$ represent the grouped miR-144-5p, miR-4732-5p and miR-451a. miR, microRNA; SE, standard error; OR, odds ratio; CI, confidence interval.

Table VI. Results of enrichment analysis for predicted targets of selected miRNAs.

\begin{tabular}{|c|c|c|c|}
\hline miRNA & GO term & Description & P-value \\
\hline \multirow[t]{5}{*}{ hsa-miR-144-3p } & 0045449 & Regulation of transcription & 0.0057 \\
\hline & 0032989 & Cellular component morphogenesis & 0.0022 \\
\hline & 0000902 & Cell morphogenesis & 0.0047 \\
\hline & 0014706 & Striated muscle tissue development & 0.0001 \\
\hline & 0060537 & Muscle tissue development & 0.0001 \\
\hline \multirow[t]{5}{*}{ hsa-miR-144-5p } & 0045449 & Regulation of transcription & 0.0016 \\
\hline & 0006350 & Transcription & 0.0001 \\
\hline & 0051252 & Regulation of RNA metabolic process & 0.0043 \\
\hline & 0006355 & Regulation of transcription, DNA-dependent & 0.0071 \\
\hline & 0010604 & Positive regulation of macromolecule metabolic processes & 0.0001 \\
\hline hsa-miR-451a & 0042127 & Regulation of cell proliferation & 0.0034 \\
\hline
\end{tabular}

miRNA, microRNA; GO, gene ontology.

hsa-miR-4732-5p, as identified in the miRBase database (www.mirbase.org/cgi-bin/mirna_entry.pl?acc=MI0000460).

This miRNA cluster is highly conserved across different types of species (http://genome.ucsc.edu/). In addition, members of the miR-144/451 cluster are located in the same intronic region between FLOT2 and FAM222B, which is 361 base pairs in length; therefore, these miRNA sequences may share common promoters. A study by Jiang et al (25) reported that the overexpression of miR-144-3p, miR-144-5p and miR-451a promoted pancreatic cell proliferation by targeting the PTEN/AKT signaling pathway. In addition, Wang et al (21) demonstrated that absence of the miR-144/451 cluster activated Rac-1-mediated oxidative stress signaling in cardiovascular cells, which impaired ischemic preconditioning-mediated cardioprotection. Zhang et al (31) demonstrated that miR-144 and miR-451 individually augmented cardiomyocyte survival and mediated cooperative functions by promoting the expression of GATA-4 protein. Furthermore, reduced levels of miR-144 and miR-451 have been shown to be inversely correlated with increased expression and phosphorylation of protein kinase AMPK (32). Considering the aforementioned studies, members of the miR-144/451 cluster may serve important roles in numerous biological pathways and may potentially be used as biomarkers for diagnosis or therapy of cancer. However, to date, the majority of studies involving the miR-144/451 cluster have focused on investigating the functional roles of individual miRNA members (33-45).
Aberrant expression of miR-451a has been observed in lung, stomach and breast cancer, in addition to glioma and leukemia (33-36). Fukumoto et al (33) reported that miR-451a inhibits the invasion and migration of hypopharyngeal squamous cell carcinoma cells by activating ESDN and DCBLD2. In addition, circulating miR-451a was demonstrated to be a potential biomarker for the diagnosis of papillary thyroid carcinoma (37). Through MIF signaling pathway, miR-451a enhanced tamoxifen sensitivity and inhibited the proliferation of breast cancer cells (38). Furthermore, miR-144-3p and miR-144-5p, encoded by the miR-144 gene, were downregulated in a variety of tumor tissues and cells, and their overexpression was correlated with poor outcome in several human cancer types (39-41). miR-144-3p has been shown to inhibit the expression of ZEB1 and ZEB2, thereby promoting the expression of cadherin and inhibiting cell invasion (42). Guo et al (43) demonstrated that miR-144 inhibits the expression of E2H2, thus affecting the Wnt/ $\beta$-catenin signaling pathway and cell proliferation. A study by Matsushita et al (44) demonstrated that miR-144-5p targets CCNE1/2 directly to suppress the proliferation of bladder cancer cells. The miR-4732-3p and miR-4732-5p members of the miR-144/451 cluster were identified after the other members, and therefore, few studies currently exist about these miRNAs $(45,46)$. Omura et al (45) suggested that miR-4732-5p may facilitate the prediction of disease recurrence following S-1 chemotherapy treatment through mutational analysis and sequence similarity. 


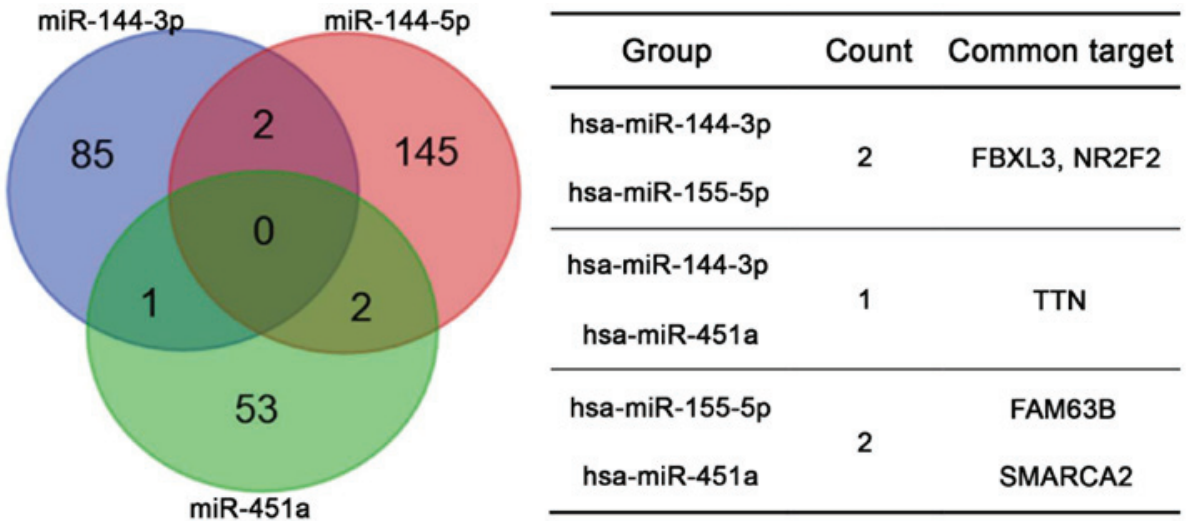

Figure 2. Identification and interaction of the potential targets of hsa-miR-144-3p, hsa-miR-144-5p and hsa-miR-451a. The Venn diagram displays the potential targets as predicted by TargetScan, miRDB, PicTar and miRanda algorithms. The number and identity of common targets are shown in the table. miR, microRNA.

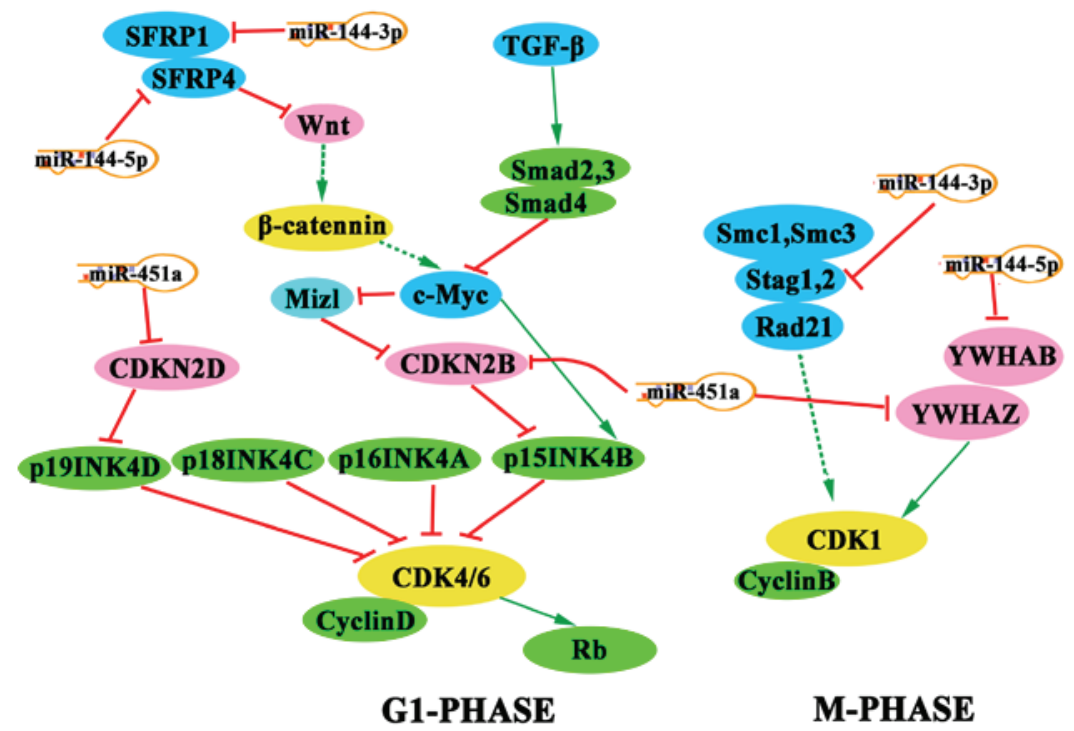

Figure 3. Pathway analysis of predicted targets of miR-144-3p, miR-144-5p and miR-451a. Pathway analysis was conducted using the Kyoto Encyclopedia of Genes and Genomes database. Solid lines indicate a direct effect, while dotted lines indicate an indirect effect. miR, microRNA.

Furthermore, Pouladi et al (46) demonstrated that, by binding with the 5'-untranslated region of WRAP53, hsa-miR-4732-5p promoted breast cancer progression. Ultimately, these studies provide evidence demonstrating that members of the miR-144/451 cluster appear to serve important roles in multiple biological pathways, and that individual miRNAs of the miR-144/451 cluster may function as part of different biological pathways in different types of tissues.

A notable finding of the present study was that the expression levels of all five members of the miR-144/451 cluster were associated with each other, particularly miR-144-5p, miR-4732-5p and miR451a. Thus, it is possible that members of the miR-144/451 cluster are expressed from the same primary transcript. By conducting a search of the NCBI database (http://www.ncbi.nlm.nih.gov/), sequences encoding miR-144-5p, miR-4732-5p and miR-451a were found to be located at the 5 '-end of precursors, indicating that they may share the same transcription and regulatory processes, which may enable an improved understanding of the expression patterns of the miR-144/451 cluster. In addition, low expression levels of hsa-miR-144-3p and hsa-miR-144-5p were found to be associated with an increased risk for esophageal carcinoma. Thus, hsa-miR-144-3p and hsa-miR-144-5p may function together and be a more stable and reliable biomarker for the early screening of high-risk populations for early diagnosis.

Previous studies have demonstrated that clustered miRNAs may mediate cooperative functions by targeting the same or multiple genes simultaneously $(15,16,47)$. Using bioinformatics analyses, the potential common or similar molecular functions of miR-144-3p, miR-144-5p and miR-451a, which are aberrantly expressed in tumor tissues, were explored in the current study. KEGG pathway analysis also demonstrated that miR-451a suppresses CDK4/6 by targeting CDKN2D and CDKN2B during G1-phase, which may induce G1 arrest. Notably, Zang et al (22) validated these findings in the EC9706 esophageal carcinoma cell line, whereby miR-451 arrested cells in G1 phase by targeting CDKN2D. The results of the present study suggest that miR-144-3p, miR-144-5p and miR-451a may disrupt the expression of CDK1 by inhibiting the activity of STAG1, YWHAB and YWHAZ, respectively. 
In addition, miR-144-3p and miR-144-5p participate in the Wnt signaling pathway by inhibiting SFRP1 and SFRP4, respectively, and the G1-phase may be affected through interference with the activity of c-Myc. The members of the miR-144/451 cluster mediate synergistic regulatory effects on the cell cycle during different phases, and therefore may present potential biomarkers for the early diagnosis of esophageal carcinoma. The miR-144/451 cluster appears to be more sensitive than a single miRNA, as it provides more comprehensive information. By promoting the expression of miR-144/451 cluster members, it is hypothesized that relative targets will be regulated together, which will help to eliminate resistance generated by targeting a single miRNA of miR-144/451 cluster. Considering the limitations of bioinformatics prediction and the complexity of biological regulatory networks, further experimental studies should be conducted to validate the regulatory mechanisms of the miR-144/451 cluster in esophageal carcinoma.

In conclusion, the present study provided evidence demonstrating that clustered miRNAs encoded by miR-144/451 gene can interact with a number of tumor-associated factors. The expression levels of miR-144/451 cluster members were downregulated in esophageal carcinoma tissues compared with adjacent non-tumor tissues. Notably, the expression levels of all five miR-144/451 cluster members were found to be associated with each other. In addition, downregulation of hsa-miR-144-3p and hsa-miR-144-5p was a potential risk factor for esophageal carcinoma development. hsa-miR-144-3p and hsa-miR-144-5p, representing the miR-144/451 cluster, may serve as potential biomarkers for the early detection of esophageal carcinoma. Further bioinformatics analyses indicated that members of the miR-144/451 cluster may function together in the progression of esophageal carcinoma. However, these molecular mechanisms remain to be verified by further experimental studies. More detailed understanding of the miR-144/451 cluster may provide new diagnostic and therapeutic approaches for patients with esophageal carcinoma.

\section{Acknowledgements}

The present study was supported by the National Natural Science Foundation of China (grant nos. 81172747, 81573108 and 81573191), and the New Century Excellent Talents in University from the Ministry of Education (grant no. NCET-13-0124).

\section{References}

1. Stewart W and Wild P: World Cancer Report 2014. IARC Press: Lyon, France, 2015.

2. Jemal A, Bray F, Center MM, Ferlay J, Ward E and Forman D: Global cancer statistics. CA Cancer J Clin 61: 69-90, 2011.

3. Shadfan A, Hellebust A, Richards-Kortum R and Tkaczyk T: Confocal foveated endomicroscope for the detection of esophageal carcinoma. Biomed Opt Express 6: 2311-2324, 2015.

4. Wang S, Du Z, Luo J, Wang X, Li H, Liu Y, Zhang Y, Ma J, Xiao W, Wang Y and Zhong X: Inhibition of heat shock protein 90 suppresses squamous carcinogenic progression in a mouse model of esophageal cancer. J Cancer Res Clin Oncol 141: 1405-1416, 2015.

5. Reinhart BJ, Slack FJ, Basson M, Pasquinelli AE, Bettinger JC, Rougvie AE, Horvitz HR and Ruvkun G: The 21-nucleotide let-7 RNA regulates developmental timing in Caenorhabditis elegans. Nature 403: 901-906, 2000.
6. Lagos-Quintana M, Rauhut R, Lendeckel W and Tuschl T: Identification of novel genes coding for small expressed RNAs. Science 294: 853-858, 2001.

7. Yue J and Tigyi G: Conservation of miR-15a/16-1 and miR-15b/16-2 clusters. Mamm Genome 21: 88-94, 2010.

8. Chan WC, Ho MR, Li SC, Tsai KW, Lai CH, Hsu CN and Lin WC: MetaMirClust: Discovery of miRNA cluster patterns using a data-mining approach. Genomics 100: 141-148, 2012.

9. Olive V, Li Q and He L: miR-17-92: A polycistronic oncomir with pleiotropic functions. Immunol Rev 253: 158-166, 2013.

10. Mohan S, Wergedal JE, Das S and Kesavan C: Conditional disruption of miR17-92 cluster in collagen type I-producing osteoblasts results in reduced periosteal bone formation and bone anabolic response to exercise. Physiol Genomics 47: 33-43, 2015.

11. Luo T, Cui S, Bian C and Yu X: Crosstalk between TGF- $\beta /$ Smad3 and BMP/BMPR2 signaling pathways via miR-17-92 cluster in carotid artery restenosis. Mol Cell Biochem 389: 169-176, 2014.

12. Brockway S and Zeleznik-Le NJ: WEE1 is a validated target of the microRNA miR-17-92 cluster in leukemia. Cancer Genet 208: 279-287, 2015.

13. Bazot Q, Paschos K, Skalska L, Kalchschmidt JS, Parker GA and Allday MJ: Epstein-barr virus proteins EBNA3A and EBNA3C together induce expression of the oncogenic MicroRNA cluster miR-221/miR-222 and ablate expression of its target p57KIP2. PLoS Pathog 11: e1005031, 2015.

14. Zhu H, Han C, Lu D and Wu T: miR-17-92 cluster promotes cholangiocarcinoma growth: Evidence for PTEN as downstream target and IL-6/Stat3 as upstream activator. Am J Pathol 184: 2828-2839, 2014

15. Besser J, Malan D, Wystub K, Bachmann A, Wietelmann A, Sasse P,Fleischmann BK, Braun T and Boettger T: MiRNA-1/133a clusters regulate adrenergic control of cardiac repolarization. PloS One 9: e113449, 2014.

16. Gits CM, van Kuijk PF, Jonkers MB, Boersma AW, Smid M, van Ijcken WF, Coindre JM, Chibon F, Verhoef C, Mathijssen RH, et al: MicroRNA expression profiles distinguish liposarcoma subtypes and implicate miR-145 and miR-451 as tumor suppressors. Int J Cancer 135: 348-361, 2014.

17. Li Y, Li W, Ying Z, Tian H, Zhu X, Li J and Li M: Metastatic heterogeneity of breast cancer cells is associated with expression of a heterogeneous TGF $\beta$-activating miR424-503 gene cluster. Cancer Res 74: 6107-6118, 2014.

18. Wystub K, Besser J, Bachmann A, Boettger T and Braun T: miR-1/133a clusters cooperatively specify the cardiomyogenic lineage by adjustment of myocardin levels during embryonic heart development. PLoS Genet 9: e1003793, 2013.

19. Yang M, Liu R, Sheng J, Liao J, Wang Y, Pan E, Guo W, Pu Y and Yin L: Differential expression profiles of microRNAs as potential biomarkers for the early diagnosis of esophageal squamous cell carcinoma. Oncol Rep 29: 169-176, 2013.

20. Liu L, Wang S, Chen R, Wu Y, Zhang B, Huang S, Zhang J, Xiao F, Wang $M$ and Liang Y: Myc induced miR-144/451 contributes to the acquired imatinib resistance in chronic myelogenous leukemia cell K562. Biochem Biophys Res Commun 425: 368-373, 2012.

21. Wang X, Zhu H, Zhang X, Liu Y, Chen J, Medvedovic M, Li H, Weiss MJ, Ren X and Fan GC: Loss of the miR-144/451 cluster impairs ischaemic preconditioning-mediated cardioprotection by targeting Rac-1. Cardiovasc Res 94: 379-390, 2012.

22. Zang WQ, Yang X, Wang T, Wang YY, Du YW, Chen XN, Li M and Zhao GQ: MiR-451 inhibits proliferation of esophageal carcinoma cell line EC9706 by targeting CDKN2D and MAP3K1. World J Gastroenterol 21: 5867-5876, 2015.

23. Wang T, Zang WQ, Li M, Wang N, Zheng YL and Zhao GQ: Effect of miR-451 on the biological behavior of the esophageal carcinoma cell line EC9706. Dig Dis Sci 58: 706-714, 2013.

24. Xie Z, Chen G, Zhang X, Li D, Huang J, Yang C, Zhang P, Qin Y, Duan Y, Gong B and Li Z: Salivary microRNAs as promising biomarkers for detection of esophageal cancer. PloS One 8: e57502, 2013

25. Jiang X, Shan A, Su Y, Cheng Y, Gu W, Wang W, Ning G and Cao Y: miR-144/451 promote cell proliferation via targeting PTEN/AKT pathway in insulinomas. Endocrinology 156: 2429-2439, 2015.

26. Rice TW, Blackstone EH and Rusch VW: 7th Edition of the AJCC cancer staging manual: Esophagus and esophagogastric junction. Ann Surg Oncol 17: 1721-1724, 2010.

27. Livak KJ and Schmittgen TD: Analysis of relative gene expression data using real-time quantitative PCR and the 2(-Delta Delta C(T)). Method 25: 402-408, 2001. 
28. Lee Y, Kim M, Han J, Yeom KH, Lee S, Baek SH and Kim VN: MicroRNA genes are transcribed by RNA polymerase II. EMBO J 23: 4051-4060, 2004.

29. Yu J, Wang F, Yang GH, Wang FL, Ma YN, Du ZW and Zhang JW: Human microRNA clusters: Genomic organization and expression profile in leukemia cell lines. Biochem Biophys Res Commun 349: 59-68, 2006.

30. Tian Y, Pan Q, Shang Y, Zhu R, Ye J, Liu Y, Zhong X, Li S, He Y, Chen L, et al: MicroRNA-200 (miR-200) cluster regulation by achaete scute-like 2 (Ascl2): Impact on the epithelial-mesenchymal transition in colon cancer cells. J Biol Chem 289: 36101-36115, 2014.

31. Zhang X, Wang X, Zhu H, Zhu C, Wang Y, Pu WT, Jegga AG and Fan GC: Synergistic effects of the GATA-4-mediated miR-144/451 cluster in protection against simulated ischemia/reperfusion-induced cardiomyocyte death. J Mol Cell Cardiol 49: 841-850, 2010.

32. Turczynska KM, Bhattachariya A, Säll J, Göransson O, Swärd K, Hellstrand P and Albinsson S: Stretch-sensitive down-regulation of the miR-144/451 cluster in vascular smooth muscle and its role in AMP-activated protein kinase signaling. PloS One 8: e65135, 2013.

33. Fukumoto I, Kinoshita T, Hanazawa T, Kikkawa N, Chiyomaru T, Enokida H, Yamamoto N, Goto Y, Nishikawa R, Nakagawa M, et al: Identification of tumour suppressive microRNA-451a in hypopharyngeal squamous cell carcinoma based on microRNA expression signature. Br J Cancer 111: 386-394, 2014.

34. Moreira FC, Assumpção M, Hamoy IG, Darnet S, Burbano R, Khayat A, Gonçalves AN, Alencar DO, Cruz A, Magalhães L, et al: MiRNA expression profile for the human gastric antrum region using ultra-deep sequencing. PloS One 9: e92300, 2014.

35. Ouyang M, Li Y, Ye S, Ma J, Lu L, Lv W, Chang G, Li X, Li Q, Wang $S$ and Wang W: MicroRNA profiling implies new markers of chemoresistance of triple-negative breast cancer. PloS One 9: e96228, 2014.

36. Babapoor S, Fleming E, Wu R and Dadras SS: A novel miR-451a isomiR, associated with amelanotypic phenotype, acts as a tumor suppressor in melanoma by retarding cell migration and invasion. PloS One 9: e107502, 2014.

37. Li M, Song Q, Li H, Lou Y and Wang L: Circulating miR-25-3p and miR-45la may be potential biomarkers for the diagnosis of papillary thyroid carcinoma. PloS One 10: e0132403, 2015.
38. Liu Z, Miao T, Feng T, Jiang Z, Li M, Zhou L and Li H: miR-451a inhibited cell proliferation and enhanced tamoxifen sensitive in breast cancer via macrophage migration inhibitory factor. Biomed Res Int 2015: 207684, 2015.

39. Katsuura S, Kuwano Y, Yamagishi N, Kurokawa K, Kajita K, Akaike Y, Nishida K, Masuda K, Tanahashi T and Rokutan K: MicroRNAs miR-144/144* and miR-16 in peripheral blood are potential biomarkers for naturalistic stress in healthy Japanese medical students. Neurosci Lett 516: 79-84, 2012.

40. Keller A, Leidinger P, Vogel B, Backes C, ElSharawy A, Galata V, Mueller SC, Marquart S, Schrauder MG, Strick R, et al: miRNAs can be generally associated with human pathologies as exemplified for miR-144. BMC Med 12: 224, 2014.

41. Chen S, Li P, Li J, Wang Y, Du Y, Chen X, Zang W, Wang H, Chu H, Zhao G and Zhang G: MiR-144 inhibits proliferation and induces apoptosis and autophagy in lung cancer cells by targeting TIGAR. Cell Physiol Biochem 35: 997-1007, 2015.

42. Guan H, Liang W, Xie Z, Li H, Liu J, Liu L, Xiu L and Li Y: Down-regulation of miR-144 promotes thyroid cancer cell invasion by targeting ZEB1 and ZEB2. Endocrine 48: 566-574, 2015.

43. Guo Y, Ying L, Tian Y, Yang P, Zhu Y, Wang Z, Qiu F and Lin J: miR-144 downregulation increases bladder cancer cell proliferation by targeting EZH2 and regulating Wnt signaling. FEBS J 280: 4531-4538, 2013.

44. Matsushita R, Seki N, Chiyomaru T, Inoguchi S, Ishihara T, Goto Y, Nishikawa R, Mataki H, Tatarano S, Itesako T, et al: Tumour-suppressive microRNA-144-5p directly targets CCNE1/2 as potential prognostic markers in bladder cancer. Br J Cancer 113: 282-289, 2015.

45. Omura T, Shimada Y, Nagata T, Okumura T, Fukuoka J, Yamagishi F, Tajika S, Nakajima S, Kawabe A and Tsukada K: Relapse-associated microRNA in gastric cancer patients after S-1 adjuvant chemotherapy. Oncol Rep 31: 613-618, 2014.

46. Pouladi N, Kouhsari SM,Feizi MH, Gavgani RR and Azarfam P: Overlapping region of p53/Wrap53 transcripts: Mutational analysis and sequence similarity with microRNA-4732-5p. Asian Pac J Cancer Prev 14: 3503-3507, 2013.

47. Wu J, Bao J, Kim M, Yuan S, Tang C, Zheng H, Mastick GS, $\mathrm{Xu} \mathrm{C}$ and Yan W: Two miRNA clusters, miR-34b/c and miR-449, are essential for normal brain development, motile ciliogenesis, and spermatogenesis. Proc Natl Acad Sci USA 111: E2851-E2857, 2014. 